\title{
A proposal for a tailored protocol for focal suppurative infection of the central nervous system: analysis of an institutional experience in pediatric patients
}

\author{
Suhas Udayakumaran, MCh, and Ticini Joseph, DNB(Neurosurgery) \\ Division of Paediatric Neurosurgery, Amrita Institute of Medical Sciences and Research Centre, Kochi, Kerala, India
}

\begin{abstract}
OBJECTIVE The incidence of focal suppurative infections (FSIs) of the brain has significantly decreased owing to the better health and fundamental conditions of the population on the one hand and earlier detection and the availability of more potent antibiotics on the other. Interestingly, the antibiotic protocols have not been well defined in terms of duration despite a prompter diagnosis, definitive management of the etiology, and the advent of various higher-generation antibiotics. In this study, the authors evaluated the current treatment protocol. Their aim was to optimize management protocols for FSIs of the central nervous system based on clinical parameters.
\end{abstract}

METHODS The study was a retrospective analysis of all children who had undergone surgical management for an FSI at the Division of Paediatric Neurosurgery, Amrita Institute of Medical Sciences and Research Centre, Kochi, Kerala, in the period from January 2001 to February 2018. In addition to demographic characteristics, the details of culture reports and antibiotic regimens were collected. The response to treatment was compared to changes in the infective profile (Creactive protein) and imaging. Instances of reaspiration were compared with clinical signs, imaging findings, and infective profiles. Treatment response was separated into two groups: responders within or at 2 weeks and responders beyond 2 weeks. The clinical characteristics of these two groups were compared.

RESULTS Forty-eight children were treated in the study period. Nineteen patients benefited from the 2-week (shortterm) protocol of intravenous antibiotics. Twenty-nine patients required more than 2 weeks (approximately 4 weeks; long-term protocol) for resolution. Of those requiring more than 2 weeks, $69 \%$ had cardiogenic etiology. All patients were followed up with a minimum of 3 weeks of oral antibiotics. In a comparative analysis between short-term and long-term responders, only etiology was significantly different. None of the patients who had the short-term protocol had a recurrence.

CONCLUSIONS A shorter antibiotic protocol can be used successfully in carefully selected patients who are surgically treated and followed up. It is clear that the 2-week intravenous antibiotic protocol is more suitable for immunocompetent patients who have a noncardiogenic etiology.

https://thejns.org/doi/abs/10.3171/2019.5.FOCUS19277

KEYWORDS cerebral abscess; brain infection; brain abscess; antibiotic protocol

$\mathrm{T}$ HE incidence of focal suppurative infections (FSIs) of the central nervous system (CNS) has significantly decreased owing to the better health and fundamental conditions of the population on the one hand and earlier detection and the availability of more potent antibiotics on the other. Advances in detection and management have made it possible to diagnose these infections earlier and to hit them aggressively with a higher margin of safety.

However, the antibiotic protocols have not been well defined and have remained traditional in terms of duration despite prompter diagnosis and the advent of various higher-generation antibiotics. ${ }^{18}$ In this retrospective study of the experience with our protocol, we evaluated the cur-

ABBREVIATIONS $\mathrm{CHD}=$ congenital heart disease; $\mathrm{CNS}$ = central nervous system; $\mathrm{CRP}=\mathrm{C}$-reactive protein; $\mathrm{FSI}$ = focal suppurative infection; $\mathrm{HBOT}=$ hyperbaric oxygen therapy; WBC = white blood cell.

SUBMITTED April 1, 2019. ACCEPTED May 9, 2019.

INCLUDE WHEN CITING DOI: 10.3171/2019.5.FOCUS19277. 
rent treatment vis-à-vis specifically the antibiotics protocol. Our aim was to optimize management protocols for FSIs of the CNS based on clinical parameters.

\section{Methods}

This study was a retrospective analysis of all pediatric patients who had undergone surgical management for an FSI at the Division of Paediatric Neurosurgery, Amrita Institute of Medical Sciences and Research Centre, Kochi, Kerala, in the period from January 2001 to February 2018. Only patients younger than 15 years old were included in the study. The demographic details of the patients, clinical and infective profiles, location of the lesion, and imaging and surgical management details were entered in an Excel spreadsheet. The details of culture reports and antibiotic regimens were collected. The response to treatment was compared to changes in the infective profile (C-reactive protein $[\mathrm{CRP}]$ and white blood cell [WBC] count) and imaging studies. Imaging was repeated every 2 weeks during treatment. Additional MRI, including T2 and contrast sequences, may be indicated if a patient shows an unsatisfactory response or new clinical indications, such as an adverse infective profile (e.g., increased CRP). Instances of reaspiration were compared to clinical signs, imaging findings, and the infective profile.

Treatment response was distinguished according to time: response within or at 2 weeks (short-term protocol) and response beyond 2 weeks (long-term protocol). The clinical characteristics of the two response groups were compared in terms of age, etiology, lesion size, organism, and outcome. Details of adjuvant therapy in the form of steroids and hyperbaric oxygen therapy (HBOT) were also noted. Follow-up records were gathered, and serial imaging and clinical details were also collected. Our follow-up protocol involves repeat imaging at 3 weeks on satisfactory completion of antibiotics and then monthly for at least 3 months thereafter or until the abscess is insignificant in size or remains stable and the infective profile is insignificant.

\section{Indications for Surgery}

The following are indications for surgery: 1) mass effect with significant neurological deficit, 2) clinical or radiological progression or nonresponse on appropriate conservative management, and any diagnostic uncertainty about the lesion.

\section{Criteria for a Positive Response}

The clinical criteria for a positive response are 1) improving neurological symptoms and signs, and 2) no significant hyperthermia $\left(100^{\circ} \mathrm{F}\right.$ or $\left.38^{\circ} \mathrm{C}\right)$. The laboratory criteria for a positive response consist of 1) normal WBC counts, and 2) normal CRP and other inflammatory markers. Follow-up values are collected even after the decision has been made to stop the antibiotics and declare a cure. The MRI protocol at all stages essentially involves T2 and contrast-enhanced sequences, and response to treatment is demonstrated by stable or decreasing radiological signs with no active signs. 1) Decreasing active signs specifically pertain to reduced perilesional edema (T2 sequence) around the index FSI, reduced nonspecific signs including no new satellite lesion, and decreasing features of effacement, as well as 2) a decreasing lesion size. Note that a comparison of contrast enhancement on pretreatment and posttreatment images was not a criterion for the demonstration of a response, as discussed below. Follow-up must show clinical quiescence with decreasing signs on MRI, as described in the previous criteria. The size of enhancement is followed, but a change in the enhancement character is not an essential requirement.

All four types of criteria-clinical, laboratory, imaging, and follow-up-are essential and should be satisfied in declaring a cure of the FSI.

\section{Criteria for Stopping Intravenous Antibiotics at Two Weeks}

Even if the clinical, laboratory, and imaging criteria are satisfied, the infective profile may reach baseline values in a delayed manner. Hence, such cases are followed up until they are normalized. We have observed that the infective profile may not always reach the true normal range at 2 weeks but may show a significant decrease to near normal. This has been enough justification for us to stop antibiotics. Any need for reaspiration in the same period is considered an indication to continue the antibiotic regimen at least 2 weeks beyond the reaspiration. Even if the reaspirated material shows no growth on culture, we prefer to continue the drugs for an additional 2 weeks. Our reasoning for this decision is as follows: 1) The culture may be negative as the patient was on a sensitive antibiotic. 2) Despite being on sensitive antibiotics, a patient's response may be slow or inadequate as reflected by the need for reaspiration and hence the need to continue antibiotics.

\section{Decision-Making Algorithm}

Initially, a broad-spectrum antibiotic is chosen, which is later switched to a specific antibiotic based on the culture report or molecular diagnosis (which was recently added to our protocol for a culture-negative abscess but was not analyzed for this study; Fig. 1). If the culture report is negative for an organism, the broad-spectrum antibiotic is continued. A baseline infective profile along with frequent repetition of the same is a key element of our decisionmaking and ultimately guides the duration of antibiotics.

\section{Definitions Used in This Study}

Cure of an abscess was considered as such when the four types of criteria for a positive response were satisfied. Nonresponse was defined as an increase in the size of an abscess on imaging and clinical findings suggesting progression while on antibiotics for at least 2 weeks or even earlier if there were clinical concerns. Recurrence was defined as an increase in the size of an abscess on imaging with or without clinical findings suggesting a progression, after successful completion of antibiotics and a documented decrease in the size of the abscess after 3 months of follow-up.

\section{Statistical Analysis}

Correlative analysis was performed in terms of the presentation and etiology of an abscess, mode of surgical treatment, duration of antibiotics, and outcome. The sen- 


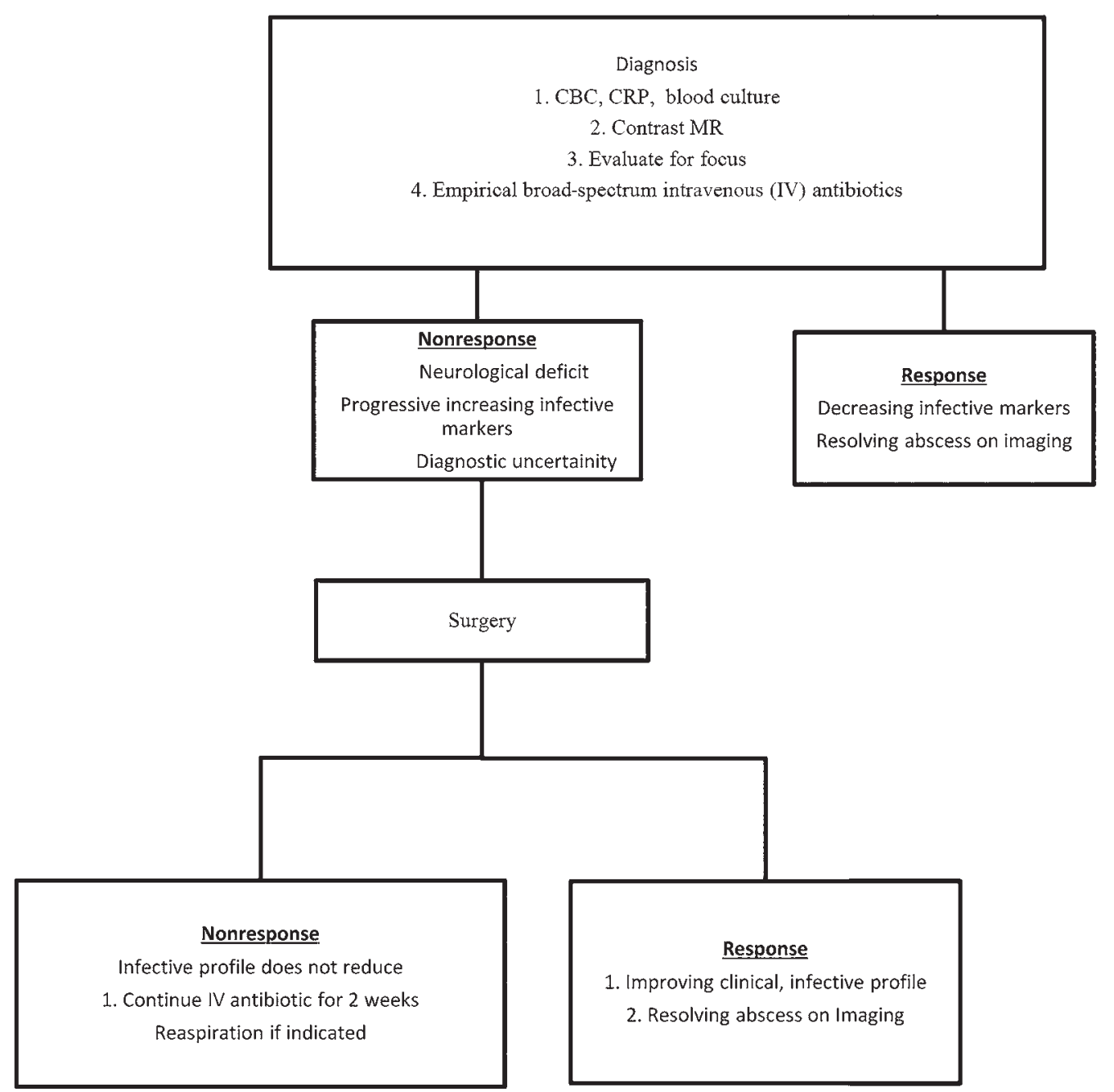

FIG. 1. Decision-making algorithm. Initially, a broad-spectrum antibiotic is chosen, which is later switched to a specific antibiotic based on the culture report or molecular diagnosis. If the culture report is negative for an organism, the broad-spectrum antibiotic is continued. A baseline infective profile along with frequent repetition of the same is a key element of our decision-making and ultimately guides the duration of antibiotics.

sitivity of an infective profile as a guide for a response to treatment was analyzed.

Statistical analysis was performed using IBM SPSS version 20.0 software (IBM Corp.). Data were entered into an Excel spreadsheet (Microsoft Corp.). Data validation and analysis were done using SPSS version 25.0 statistical software. Continuous variables were expressed as the mean \pm standard deviation. All categorical variables were expressed as a percentage or proportion. Comparison of normally (Gaussian) distributed continuous variables among the categories was done using the independent sample ttest. Comparison of categorical variables was performed using either the chi-square test or Fisher exact test based on the number of observations. All $p$ values $<0.05$ were considered statistically significant.

\section{Results}

Forty-eight children, with a male/female ratio of $2: 1$ $(32 / 16)$, were seen for cerebral abscess in the period be- tween January 2001 and February 2018. The age range was 27 days to 14 years (mean 9.5 years; Fig. 2A). The most common presentation was features of raised intracranial pressure (ICP) in 63\% (30) of patients. The triad of ICP features, fever, and neurological deficit was seen in only 9 patients $(18.8 \%)$. Altered sensorium was seen in $10 \%(5 / 48)$, and signs of meningeal irritation were seen in $10 \%(5 / 48)$.

The most common organism was streptococcus (21 patients), while other organisms were staphylococcus (2), Aspergillus (2), Haemophilus influenzae type B (2), enterococcus (2), Klebsiella (2), Proteus (1), Candida (1), Moraxella (1), Escherichia coli (1), Pseudomonas (1), and uncharacterized in 3 patients. Streptococcus was the most common organism isolated in a cardiogenic abscess in $84 \%$ ( 21 patients). Culture results were negative in 5 patients. Among the 16 patients requiring reaspiration, cultures from 7 still grew streptococci; hence, reaspiration was considered to be a factor in deciding to continue antibiotics. About 15 (15/48) samples including reaspira- 
Age distribution

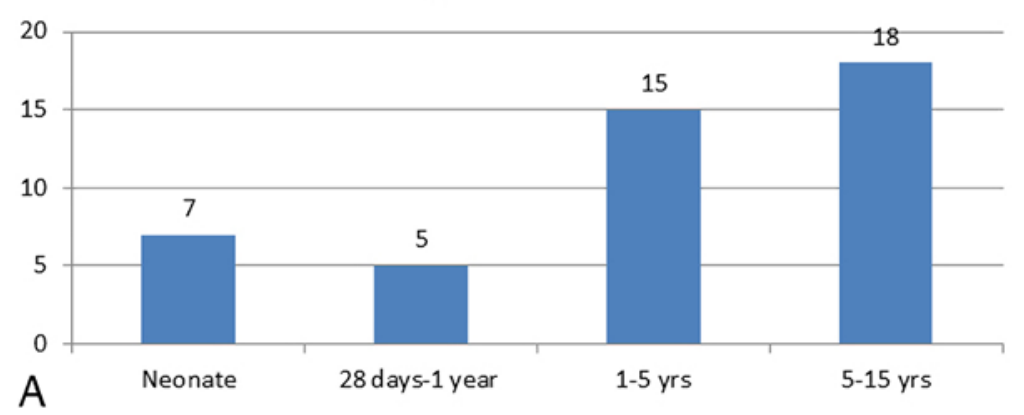

Location

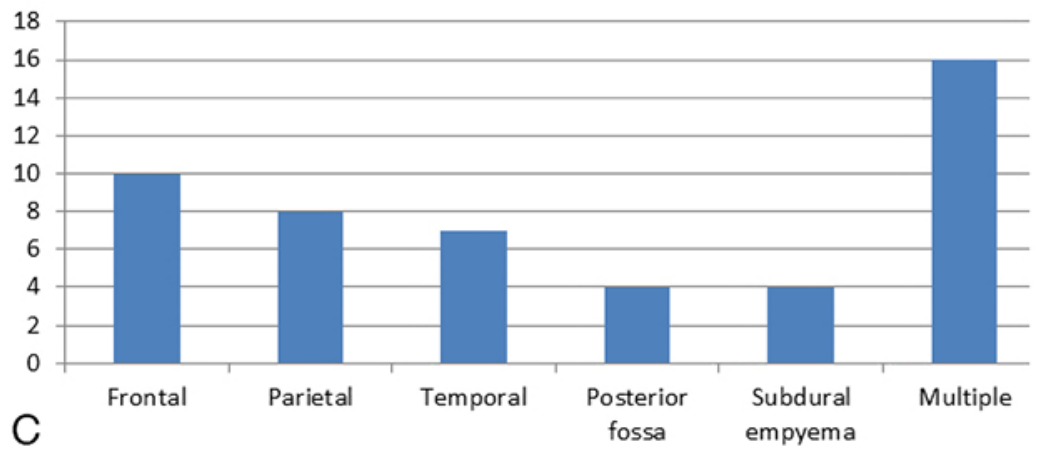

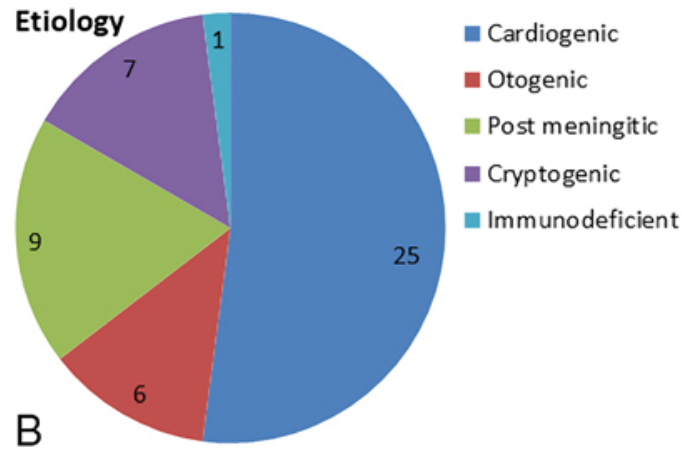

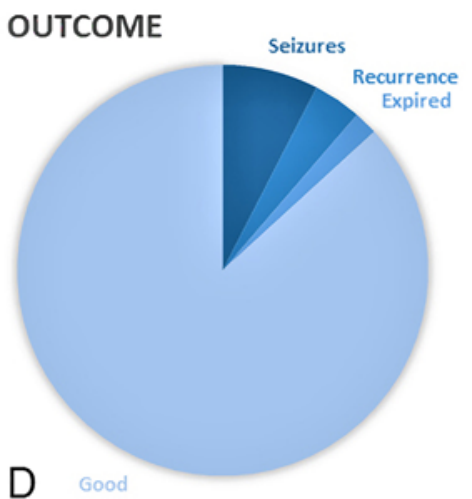

FIG. 2. A: Bar graph showing the age distribution of the patients. The youngest child was 27 days old, and the oldest was 14 years old. The $y$-axis represents the number of patients. B: Pie chart showing the distribution of etiologies. The commonest etiology was cardiogenic (25 cases), with the youngest patient an infant whose cardiac lesion was identified on evaluation of the cerebral abscess. The most common etiology in the neonatal age group was postmeningitic. The otogenic abscess was seen only after 5 years of age. C: Bar graph showing the distribution of the location of cerebral abscesses in the cohort. The most common location was frontal (10 lesions), and others were parietal (8 lesions) and temporal (7 lesions). D: Pie chart showing outcomes. In general, 46 patients had a good outcome, although 4 patients had seizures. Two (4.2\%) of the 48 patients had a recurrence; one of them died, and another did well after reaspiration. Two patients in the series died. One death was attributable to shunt infection and sepsis, which occurred 1.5 years after abscess treatment. The second death was attributable to the recurrence of posterior fossa Aspergillus abscess, and the patient became moribund in extremis. Mortality was $4 \%$ with only one death directly due to an abscess. Multiple $=$ multiple locations.

tion samples were culture negative. Organisms like $\mathrm{Mo}$ raxella and Proteus were seen in otogenic brain abscess. Aspergillus was isolated in an immunodeficient abscess (1 patient) and a postmeningitic abscess in a neonate. Enterococci (2 patients) were isolated only in a postmeningitic abscess in our series (Fig. 2B). In 2 cases, the organisms were identified in polymerase chain reaction (PCR) assays when the routine cultures were negative (i.e., organisms identified as streptococcus and Prevotella).

Nineteen patients benefited from the 2-week intravenous antibiotic protocol. Twenty-nine patients required more than 2 weeks (approximately 4 weeks) for resolution. Of those patients requiring more than 2 weeks, $69 \%$ had a cardiogenic etiology. All patients were followed up with a minimum of 3 weeks of oral antibiotics (also chosen per the sensitivity report).

Baseline CRP values were elevated in $71 \%$ of the patients (34/48). In treatment-sensitive cases, the mean reduction in CRP was found to be on average $70.19 \mathrm{mg} / \mathrm{L}$ within 2 weeks of treatment. WBC counts were significantly higher in most of the patients (40/48) with increased $\mathrm{CRP}$ and were borderline in others. The WBC trend paralleled that for CRP in a majority of cases except those with secondary infections. WBC counts can rise in a secondary infection (when CRP can also rise) and occasionally nonspecifically with unclear explanation due to other causes.

Surgical management predominantly involved burr hole aspiration (42 patients). Six patients underwent craniotomy for related reasons (recurrences and 1 dermoid). Sixteen patients underwent reaspirations within 2-5 weeks of the first surgery for a persistent abscess or an increase in the size of an abscess along with an unsatisfactory clinical response. Among these cases, 81\% (13/16) had a cardiogenic etiology. As defined in the criteria for stopping antibiotics, a patient who had responded to shortterm antibiotics did not require any reaspiration; in other words, reaspiration was a criterion for the continuation of treatment beyond 2 weeks, among others. Ten (40\%) of the 25 patients with cardiogenic abscess required reaspiration after the initial surgery, compared with 13 patients (27\%; 16 reaspirations with 3 patients requiring recurrent reaspirations) among the total cohort of 48 patients (only $7.6 \%$ with reaspiration of an abscess secondary to noncardiac causes). Three patients required more than three aspirations, all of whom underwent craniotomy and excision (all cardiogenic cases). 
In the comparative analysis between short-term responders (within or at 2 weeks) and long-term responders (beyond 2 weeks), only etiology was found to be significantly different. Forty percent (19/48) of the patients responded to drainage and the short-term antibiotic protocol. Of these patients, 74\% (14/19) had noncardiogenic etiology while 26\% (5/19) had cardiogenic etiology (Table 1). It was clear that the cardiogenic etiology was slower to respond. None of the patients with the short-term protocol had a recurrence.

More recently in the study period, we started using HBOT early in the treatment course (2 patients). The maximum follow-up in our study was 16 years, and the minimum was 4 months. In general, 46 patients $(96 \%)$ had a good outcome, while $2(4.2 \%)$ had a recurrence. One of these latter patients died, and the other patient did well after reaspiration. We have separated the patient requiring reaspiration (13/48) from those having a recurrence for this analysis. We define reaspiration as an event occurring during treatment (see Methods). Two patients in this series died. One death was attributable to shunt infection and sepsis (1.5 years after abscess treatment), and the second was attributable to the recurrence of a posterior fossa Aspergillus abscess, as the patient became moribund in extremis. Our mortality rate was $4 \%$ with only one death directly due to the abscess (Fig. 2D).

\section{Discussion}

Cerebral abscess is a common pediatric neurological problem. Despite this, management protocols have been vague and less objective. ${ }^{16}$ There has been a change in the trends for etiology, age distribution, and microbiological spectrum of brain abscesses in the recent decade. The past decade saw more of the cardiac abscess and otologic abscess from an untreated primary etiology. ${ }^{19}$ Postmeningitic brain abscess has evolved as a cause of concern in the developing world probably because of the better survival of preterm and postmeningitic babies. The management of cerebral abscess has advanced significantly over time. Advanced diagnostics, the introduction of vaccinations, the introduction of newer-generation antibiotics, and early definitive management of primary cardiac and otologic etiologies have been significant contributors to the better present-day outcomes. Nonetheless, the antibiotic protocols and their duration have not changed since antibiotic treatment has been administered for the condition. In the following sections, we discuss the factors that can influence the antibiotic protocol and support the proposal for modification.

Ear and mastoid sources are associated with the formation of an abscess at the temporal or cerebellar locations; sinus and dental infections give rise to purulent collections in the frontal lobe; and metastatic spread from distant foci in children with congenital cardiac or pulmonary right-to-left shunts commonly results in the involvement of any parenchyma area, including parietal or occipital regions..$^{10,16}$ When congenital heart disease (CHD) remains uncorrected, it becomes a nidus for bacteria to settle and a source of bacteremia. Tetralogy of Fallot and transposition of the great vessels are the most commonly cited predis-
TABLE 1. Response to antibiotic protocol according to etiology

\begin{tabular}{ccc}
\hline \multicolumn{1}{c}{ Etiology } & $\leq 2$ Wks & $>2$ Wks \\
\hline Cardiogenic & 5 & 20 \\
\hline Noncardiogenic & 14 & 9 \\
\hline
\end{tabular}

posing factors. ${ }^{10,19}$ The peak incidence is between 4 and 7 years of age, although cases of brain abscess can occur in adults with CHD. ${ }^{12}$ We encountered 52\% (25/48) of brain abscesses associated with CHD. All of them had tetralogy of Fallot physiology. Despite identification of all these potential sources, $14 \%$ of cases were classified as cryptic brain abscess for which no obvious predisposing factor was identified.

A patient's prognosis also depends on his or her underlying cardiac condition. Certain conditions in which the pathophysiological processes can be reversed are associated with an excellent prognosis, with no risk of abscess recurrence due to the condition once the underlying condition is resolved. Patients with cyanotic physiology in whom surgical correction is not possible are at lifelong risk for cerebral abscess as well as the poor prognosis of the primary cardiac condition..$^{19}$

Culture positivity for brain abscess pus specimens of $44 \%-100 \%$ has been reported in the literature. ${ }^{89}$ In order of etiological importance, the predominant organisms causing brain abscess in children are aerobic and anaerobic streptococci $(60 \%-70 \%$ of cases), gram-negative anaerobic bacilli $(20 \%-40 \%)$, Enterobacteriaceae (20\%$30 \%)$, Staphylococcus aureus (10\%-15\%), and fungi (1\%$5 \%)^{15,16}$ In children with impaired host defenses, fungal etiology or uncommon pathogens such as Toxoplasma, Nocardia, Mycobacterium, and Listeria can be identified. Multiple aerobic and anaerobic organisms are isolated in approximately one-third of patients, especially those with chronic otitis. No growth is reported from as many as 30\% of properly handled purulent specimens. As most cases are pretreated with antibiotics at presentation, there is a high possibility for regular cultures to be negative. The importance of molecular-based genetic tests for the identification of causative organisms must be emphasized and should be part of routine diagnostics.

Medical management is empirical to start with and specific after a culture report has been received. The basis for selecting the antibiotic is usually the site of the lesion, and the suspected causative organism is presumed based on previous scientific data. In vitro, antibiotic sensitivity results have shown that a cephalosporin and metronidazole regimen, which is typically used in most neurosurgery units, is a satisfactory presumptive choice of therapy. Vancomycin may be an empirical addition for a posttraumatic abscess.

The appropriate duration of antimicrobial therapy for brain abscess remains unclear. A 6- to 8-week course of, parenteral antibiotics has been traditionally recommended provided that the etiological organisms are susceptible and adequate surgical drainage is achieved. ${ }^{14}$ The basis for this traditional regimen is unclear and, to a large extent, may be unfounded. 
Unlike in recent times, in the past, there were multiple reasons for the persistence of the traditional regimen, for example, when there was a cryptic source for the cerebral abscess. A longer antibiotic regimen was necessary when the etiology could not be identified; thus, the protocol used was empirical. For instance, an otologic or a cardiogenic etiology could not be identified because of the lack of imaging or its sensitivity. A high incidence of uncorrected CHD also contributed to the use of a traditional regimen. In the past and in developing nations, the treatment for CHD was not as advanced for early diagnosis and correction, leading to a high number of uncorrected CHD cases or patients with advanced sequelae such as pulmonary hypertension and making it impossible to safely correct by current standards.

Another possible reason that the traditional regimen has persisted may be that obligate or atypical organisms were not identified. Such identification has become possible because of advanced technology such as molecular diagnostics as opposed to conventional culture. Moreover, delayed diagnosis and delayed initiation of treatment due to the unavailability of sufficient testing methods or specific antibiotics can lead to patients being treated in advanced morbid states, adding multiple confounding factors to the goal of cure and unclear treatment results of treatment.

Poorly understood imaging endpoints have also contributed to the persistence of a traditional treatment regimen. Imaging is a key adjunct for decision-making in the management of cerebral FSI. The treatment endpoints have been traditionally based on radiology, especially the enhancement. With regard to a cerebral abscess, it is well accepted that enhancement suggests a breach of the blood-brain barrier. ${ }^{4}$ An early stage of cerebritis may also show solid enhancement. ${ }^{4}$ The dose of contrast may also alter the enhancement pattern. ${ }^{4}$ We found no literature to equate enhancement to an active infection. Hence, using enhancement as a basis for a treatment goal is not well supported. Residual enhancement can remain for as long as 8 months after hospital discharge. The exact mechanism is not known, but it probably involves an alteration of the blood-brain barrier, perhaps due to the formation of a different type of small vessel. Thus, prolonged enhancement is a nonspecific response of the brain parenchyma to various types of insult. ${ }^{20}$ In the last decade, gadolinium-enhanced MRI with diffusion-weighted images and MR spectroscopy have high sensitivity and specificity, improving diagnostic efficacy and prompter diagnosis.

For all the reasons discussed above, the traditional regimen may not have enough of a basis to be followed. In selected cases with uncomplicated infection and complete surgical evacuation of a well-delineated abscess, shorter treatment courses are likely to be sufficient. ${ }^{10,11,17}$ Nonetheless, many authorities recommend 2-3 months of additional oral antimicrobial therapy to prevent relapses.?

A nonoperative management strategy for cerebral abscess can be attempted 1) for clinically stable patients who are poor candidates for surgery or who have inaccessible lesions, ${ }^{1,3,5}$ and 2) for small collections $(2 \mathrm{~cm})$ located in well-vascularized cortical areas, which are more likely to respond to antibiotics alone. Cases treated conservatively may require a more prolonged duration of treatment and close clinical and radiographic follow-up, but this aspect is beyond the scope of this article.

Burr hole aspiration is as effective as excision in the management of most purulent collections within the brain but with less morbidity., ${ }^{6,21,22}$ Treatment response can be monitored based on the clinical condition, infective profile, and serial imaging. In our cohort, the CRP values remained stable or even increased. This finding also correlated with the persistent size of the abscess on imaging or to cases that were reaspirated, respectively. Repeat CT and MRI will eventually show a decrease in the size of the abscess, disappearance of surrounding edema, and decreased enhancement ring. These improvements are usually observed within 1-4 weeks of management, but complete radiographic resolution often extends to several months of follow-up.

Late presentation and delay in the initiation of antibiotics have been correlated to poorer outcomes. Some patients are quite moribund on presentation, and despite quick multimodal treatment, they succumb to the disease. The reported mortality rates range from $7.9 \%$ to $53 \%$. In our cohort, mortality was $4.2 \%$. One patient presented with posterior fossa abscess with severe hydrocephalus and did not respond to craniotomy, drainage, and external ventricular drain for cerebrospinal fluid, while the other patient was immunodeficient.

Certain adjuvant options such as HBOT are gaining acceptance and may become part of standard treatment protocols for managing FSI. We have started adding it to our primary treatment protocol after some references to its usefulness in FSIs of the brain, although we lack sufficient experience with it to comment affirmatively on its efficacy. ${ }^{13}$ In a study by Bartek et al., the authors concluded that HBOT was associated with fewer treatment failures and reoperations and with seemingly improved long-term outcomes. They further observed that HBOT was well tolerated and safe. Prospective studies are warranted to establish the role of HBOT in the treatment of brain abscesses. ${ }^{2}$

\section{Advantages of a Short-Term Antibiotic Protocol}

We have shown that a short-term antibiotic approach can be successfully used in a selected group of patients. Selection is a continuous process based on the ongoing treatment response rather than a decision made at the outset (Fig. 1). We noticed that the noncardiogenic abscess is more responsive to the shorter protocol. We can assume that the inherent pathophysiology of the cardiogenic abscess makes it less responsive or a slow responder to treatment with the shorter protocol. This slower responsiveness is also reflected in the fact that these cardiogenic abscesses required a higher number of reaspirations.

Whenever antibiotics are used for a shorter period, it proves more economical given the reduction in the antibiotics used, the hospital stay, and the associated expenses. The associated morbidity is also reduced. The key would remain careful selection and follow-up to avoid recurrence due to partial treatment. An uncorrected etiology would predict a need for a prolonged antibiotic course and a recurrence. This is clear from our experience with cardiogenic abscess having more frequent aspirations and requiring the longer course of antibiotics. In our experience, true recurrence happens when the source of infection (shunt meningitis in our cohort) is not eliminated or is an atypical in- 

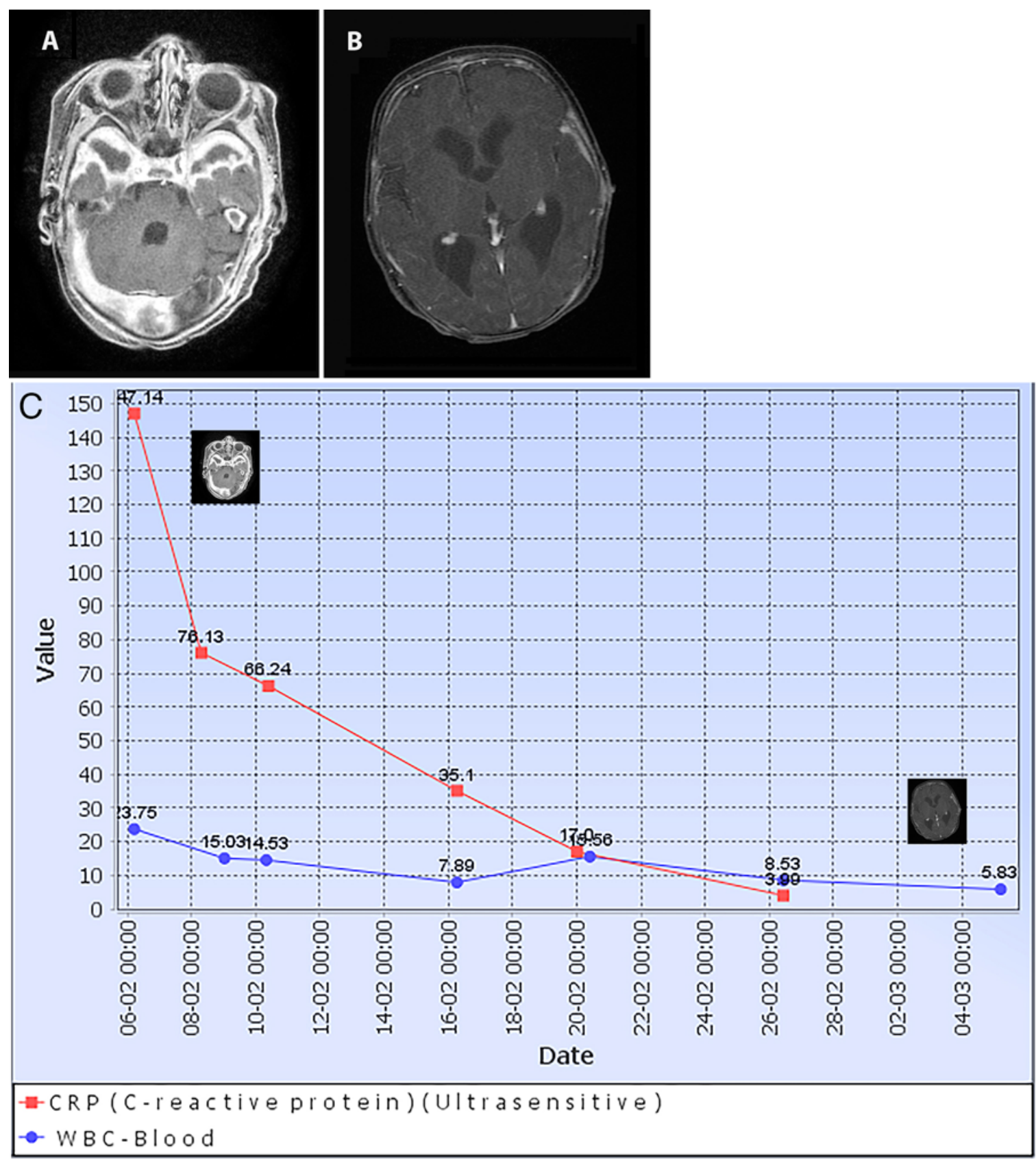

FIG. 3. A: Gadolinium-enhanced MR image of a postmeningitic subdural empyema in an infant at the beginning of treatment. Subdural aspiration and wash were performed. The culture revealed multidrug-resistant Pseudomonas sensitive only to meropenem. B: Gadolinium-enhanced MR image obtained at 4 weeks' follow-up since the diagnosis. Antibiotic was stopped at 2 weeks. Image enhancement has dramatically decreased along with a corresponding decrease in the CRP value and WBC count. We have noted that the change in the infective profile usually predates the imaging response. In our protocol, imaging is performed immediately after aspiration and then every 2 weeks to demonstrate a satisfactory response to antibiotic therapy. Additional MRI may be indicated if the patient shows an unsatisfactory response or new clinical indications, including an adverse infective profile (e.g., increased CRP). Our follow-up protocol involves repeat imaging at 3 weeks on satisfactory completion of antibiotics and then monthly for at least 3 months thereafter or until the abscess is insignificant in size or remains stable and with an insignificant infective profile. C: A falling trend in the infective profile. The antibiotics were stopped at 2 weeks. This was based on the fact that antibiotics were specific for the corresponding decreasing trend in the infective profile. The infective profile (e.g., CRP, WBC count) may reflect treatment response, inadequate response, or recurrence. Dates on the X-axis are expressed as day-month. Values on the $y$-axis are expressed in $\mathrm{mg} / \mathrm{L}$. 

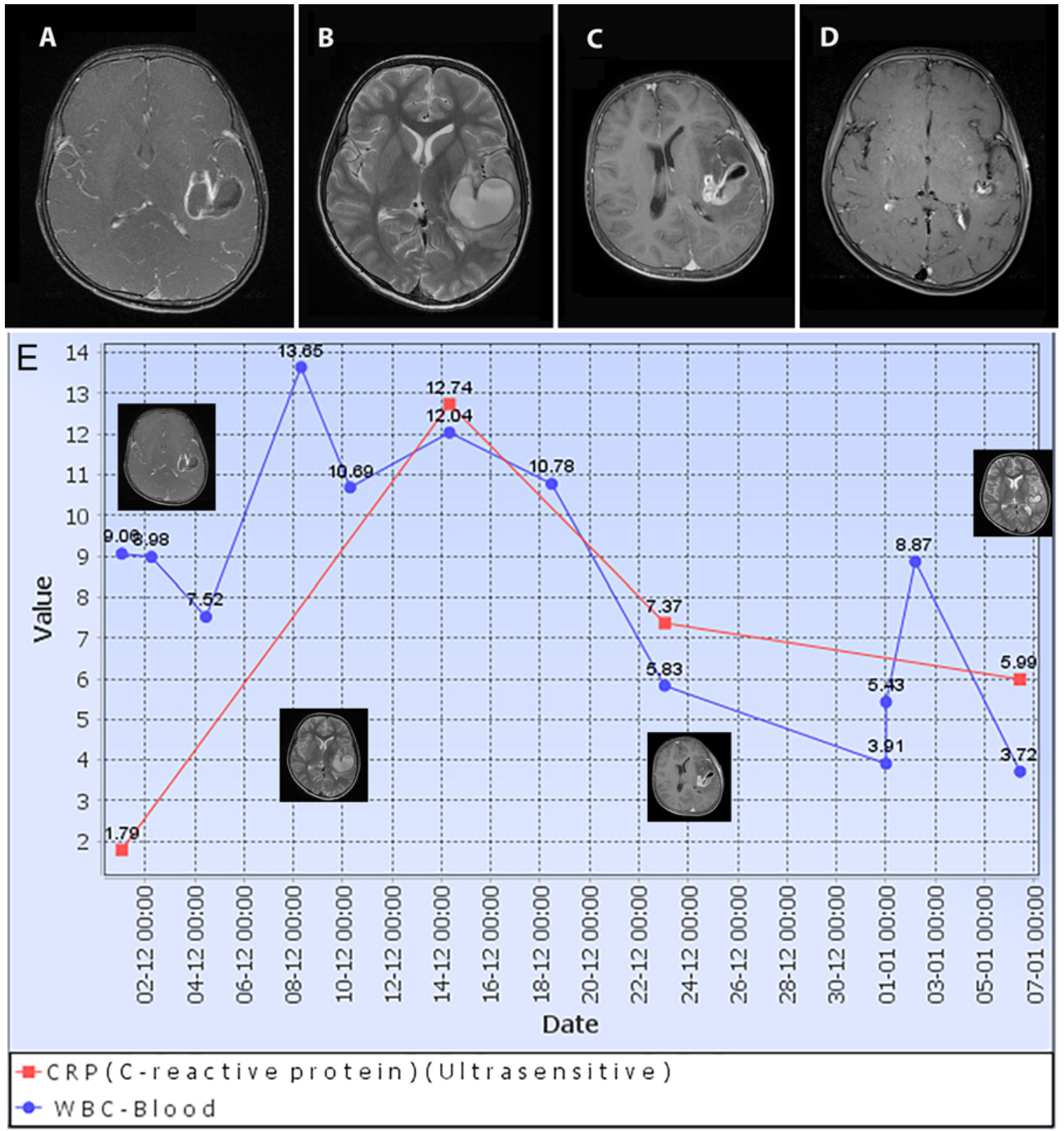

FIG. 4. A: MR image obtained at diagnosis when conservative management was initiated in a child with a cardiogenic abscess. Note the hypodense center with well-defined ring enhancement, as well as the surrounding area of edema. B: Axial T2-weighted MR image obtained when aspiration was decided on in view of the change in the infective profile (14-12 on the $x$-axis in E). Her symptoms were improving, but at this point, she complained of a new-onset headache. Only a rapid T2 protocol is used for followup during treatment. C: Gadolinium-enhanced MR image obtained when the decision was made to stop antibiotics. D: Gadolinium-enhanced MR image obtained at 4 weeks' follow-up. The corresponding trend in the infective profile is shown in panel $\mathrm{E}$. E: Changing trends in the infective profile. In the initial 2 weeks, conservative management was attempted. The child had an initial improvement in symptoms, but as CRP rose, the abscess was drained (14-12), leading to a dramatic fall in the parameters. The culture was negative, and the antibiotic was stopped at 2 weeks (30-12). Dates on the x-axis are expressed as day-month. Values on the $\mathrm{y}$-axis are expressed in $\mathrm{mg} / \mathrm{L}$. Inset images show the status at each decision.

dolent organism (e.g., Aspergillus in our cohort). This can be extrapolated from the meningitis experience wherein a 2 -week course is enough for most common organisms but not for fungal organisms or when infected implants have not been removed.

It is imperative that the antibiotic selection is appropriate. As a corollary, culture positivity may be a key selection criterion for our short-term approach. A polymicrobial and anaerobic nature of the abscess must be assumed since the likelihood of identifying an obligate anaerobe or microaerophilic is small.

Its correlation with infective markers makes following the treatment response convenient and reliable (Figs. 3 and 4). In our cohort, we had nearly $70 \%$ correlation with in- 
fective markers (CRP, WBC counts) and an equal percentage showing a responsive trend. The infective markers are an essential adjunct to our decision-making (along with radiology) to determine responsiveness to and the end of therapy. Stable and "inactive" imaging (see Methods) is an adjunct in our decision-making algorithm. Parallel clinical improvement must supplement both of these factors.

The sequelae have been noted to be minimum with aggressively treated brain abscesses. ${ }^{14}$ In our cohort, a permanent neurological deficit in the form of a focal neurological deficit was seen in only $4.2 \%$ of patients, and seizures were persistent in $8.3 \%$.

A patient's prognosis also depends on his or her underlying etiology. Certain conditions in which the pathophysiological processes can be reversed are associated with an excellent prognosis, with no risk of abscess recurrence due to the condition once that underlying condition is resolved. Patients with cyanotic physiology in whom surgical correction is not possible are at lifelong risk for cerebral abscess, in addition to the poor prognosis of the primary cardiac condition. ${ }^{19}$

\section{Limitations of the Study}

The small cohort numbers in each category for comparison of the factors did not allow us to make conclusions other than that etiology may be responsible for short-term antibiotic responsiveness. Larger numbers will be required to identify other predictors of the success of a short-term antibiotic protocol. Focal suppurative infections in adults are a different pathological entity in many ways. Our series involves a pediatric cohort; hence, we believe that a separate study is merited to analyze the applicability of our conclusion in adults with FSI.

\section{Conclusions}

An unresolved primary etiology at presentation is a risk factor for the persistence and recurrence of cerebral abscess. Biomarkers of inflammation can be used as a reliable guide for reimaging, surgical intervention during treatment, and overall response. A short course of an organismspecific antibiotic can be used successfully in carefully selected and monitored patients. It is certain that a 2 -week antibiotic protocol is more suitable for immunocompetent patients who have a noncardiogenic etiology. The prognosis of a cerebral abscess is good with minimal neurological sequelae whenever the primary etiology can be resolved and diagnosis and treatment are prompt.

\section{References}

1. Barsoum AH, Lewis HC, Cannillo KL: Nonoperative treatment of multiple brain abscesses. Surg Neurol 16:283-287, 1981

2. Bartek J Jr, Jakola AS, Skyrman S, Förander P, Alpkvist P, Schechtmann G, et al: Hyperbaric oxygen therapy in spontaneous brain abscess patients: a population-based comparative cohort study. Acta Neurochir (Wien) 158:1259-1267, 2016

3. Boom WH, Tuazon CU: Successful treatment of multiple brain abscesses with antibiotics alone. Rev Infect Dis 7:189199, 1985

4. Bradley WG Jr, Yuh WT, Bydder GM: Use of MR imaging contrast agents in the brain. J Magn Reson Imaging 3:199218,1993
5. Carpenter J, Stapleton S, Holliman R: Retrospective analysis of 49 cases of brain abscess and review of the literature. Eur J Clin Microbiol Infect Dis 26:1-11, 2007

6. Cavuşoglu H, Kaya RA, Türkmenoglu ON, Colak I, Aydin Y: Brain abscess: analysis of results in a series of 51 patients with a combined surgical and medical approach during an 11-year period. Neurosurg Focus 24(6):E9, 2008

7. Cochrane DD: Consultation with the specialist. Brain abscess. Pediatr Rev 20:209-215, 1999

8. de Louvois J: Bacteriological examination of pus from abscesses of the central nervous system. J Clin Pathol 33:6671,1980

9. de Louvois J: The bacteriology and chemotherapy of brain abscess. J Antimicrob Chemother 4:395-413, 1978

10. Fischer EG, McLennan JE, Suzuki Y: Cerebral abscess in children. Am J Dis Child 135:746-749, 1981

11. Jadavji T, Humphreys RP, Prober CG: Brain abscesses in infants and children. Pediatr Infect Dis 4:394-398, 1985

12. Kagawa M, Takeshita M, Yato S, Kitamura K: Brain abscess in congenital cyanotic heart disease. J Neurosurg 58:913917, 1983

13. Kurschel S, Mohia A, Weigl V, Eder HG: Hyperbaric oxygen therapy for the treatment of brain abscess in children. Childs Nerv Syst 22:38-42, 2006

14. Mathisen GE, Johnson JP: Brain abscess. Clin Infect Dis 25:763-781, 1997

15. Nielsen H: Cerebral abscess in children. Neuropediatrics 14:76-80, 1983

16. Sáez-Llorens X: Brain abscess in children. Semin Pediatr Infect Dis 14:108-114, 2003

17. Skoutelis AT, Gogos CA, Maraziotis TE, Bassaris HP: Management of brain abscesses with sequential intravenous/oral antibiotic therapy. Eur J Clin Microbiol Infect Dis 19:332335,2000

18. Sonneville R, Ruimy R, Benzonana N, Riffaud L, Carsin A, Tadié JM, et al: An update on bacterial brain abscess in immunocompetent patients. Clin Microbiol Infect 23:614-620, 2017

19. Udayakumaran S, Onyia CU, Kumar RK: Forgotten? Not yet. Cardiogenic brain abscess in children: a case series-based review. World Neurosurg 107:124-129, 2017

20. Whelan MA, Hilal SK: Computed tomography as a guide in the diagnosis and follow-up of brain abscesses. Radiology 135:663-671, 1980

21. Woods CR Jr: Brain abscess and other intracranial suppurative complications. Adv Pediatr Infect Dis 10:41-79, 1995

22. Yang SY: Brain abscess: a review of 400 cases. J Neurosurg 55:794-799, 1981

\section{Disclosures}

The authors report no conflict of interest concerning the materials or methods used in this study or the findings specified in this paper.

\section{Author Contributions}

Conception and design: Udayakumaran. Acquisition of data: both authors. Analysis and interpretation of data: both authors. Drafting the article: both authors. Critically revising the article: Udayakumaran. Reviewed submitted version of manuscript: Udayakumaran. Approved the final version of the manuscript on behalf of both authors: Udayakumaran. Statistical analysis: Joseph. Administrative/technical/material support: Udayakumaran. Study supervision: Udayakumaran.

\section{Correspondence}

Suhas Udayakumaran: Amrita Institute of Medical Sciences and Research Centre, Kochi, India.dr.suhas@gmail.com. 